\title{
LA VIOLENCIA CONYUGAL EN LA REPÚBLICA DOMINICANA
}

Conjugal violence in the Dominican Republic

\section{Huberto Bogaert García*}

Resumen: La violencia conyugal es un síntoma postraumático que constituye un grave problema de salud pública. La investigación realizada confirma que el sujeto masculino que fue abusado en su infancia tiene una alta probabilidad de ejercer la violencia conyugal en la adultez. El incremento de esta, dentro de la familia matrifocal dominicana, se debe a la variación del polo materno provocada por la aculturación, la emigración, la escolaridad temprana y el trabajo de la madre fuera del hogar, entre otros. En ese sentido, la agresividad suscitada por la madre y que el dispositivo matrifocal reprime, se desplaza hacia la pareja y provoca el aumento de la violencia conyugal.

Palabras clave: Violencia conyugal, abuso infantil, familia matrifocal, República Dominicana.

* Psicólogo clínico, psicoanalista y profesor del Instituto Tecnológico de Santo Domingo (INTEC). E-mail: hubertobogaert@yahoo.com 
Abstract: Conjugal violence is a post-traumatic symptom that constitutes a serious problem within the public health. This research confirms that a male who was abused as a child has a high probability of being abusive to his spouse during adulthood. The increase of conjugal violence within the Dominican matrifocal family, is due to the variation in the maternal unit caused by acculturation, migration, early childhood education, the mother's work away from home, among other causes. Accordingly, the aggression raised by the mother, which is rejected by the matrifocal family, moves towards the spouse, causing the increase of conjugal violence.

Keywords: Domestic violence, child abuse, matrifocal family, Dominican Republic.

\section{Introducción}

Este estudio sobre la violencia conyugal en la República Dominicana se ha llevado a cabo considerando tres niveles de análisis. En primer lugar, presentamos información estadística relevante sobre las víctimas y sus agresores. En segundo lugar, investigamos la asociación entre la violencia conyugal y el abuso infantil. En tercer lugar, interpretamos, desde el punto de vista etnopsicoanalítico, el origen de la violencia conyugal y su incremento en la República Dominicana, considerando la matrifocalidad de nuestra familia.

Si la matrifocalidad —o la tendencia a la matrifocalidad - condiciona la violencia conyugal y, por otra parte, la aculturación la incrementa, el recurso a un modelo de interpretación estructural etnopsicoanalítico permitiría poner de manifiesto el sentido de esos datos estadísticos desconcertantes, según los cuales los adultos jóvenes - a pesar de tener un mayor acceso a la información y a la educación- 
muestran conductas y actitudes más conservadoras. De igual modo, la interpretación estructural permitiría comprender que, de los usuarios del Centro de Intervención Conductual para Hombres que maltratan a sus parejas, el 33\% tiene estudios superiores y de postgrado, el 95\% no padece trastornos psiquiátricos, el 86\% tiene al menos un empleo, y el $62 \%$ no reporta abuso de alcohol (Vergés, 2010: 1 y 8A).

En ese sentido, el modelo que proponemos para la interpretación de la violencia conyugal permitiría superar las limitaciones, tanto del modelo estadístico, como del modelo ecológico.

\section{La violencia conyugal}

La violencia es una agresión -física, psíquica o sexual- o un daño - a la persona o a sus propiedades - que causa dolor, enfermedades, trastornos emocionales y hasta la muerte (Sonkin et al., 1985). La violencia de género es la que se ejerce contra la mujer, afectando su vida pública o privada. Cuando ocurre entre personas que tienen o han tenido una relación íntima, se denomina violencia conyugal (Heise, 1994).

La violencia conyugal puede ser física, psicológica —amenazas, gritos, aislamiento, humillaciones, destrucción de objetos, control abusivo sobre la víctima, entre otros-, sexual —coerción sexual, con o sin violencia física- o económica —control abusivo del acceso al dinero, decisiones arbitrarias sobre gastos, etc.- .

El Estado dominicano ha creado mecanismos legales e institucionales para prevenir la violencia de género. Conviene destacar la Ley de Violencia Intrafamiliar N. ${ }^{\circ} 24-97$ y el Código Procesal Penal, instrumentos que junto con la Ley 88-03 - que instituye en todo el territorio nacional las Casas de Acogida o Refugios, con sus Reglamentos- han contribuido al fortalecimiento del Sistema Judicial y a la protección de la mujer. Sin embargo, como señalan Córdoba y Pérez, aunque el país ha mostrado mejoras en 
el establecimiento de una normativa y en la instauración de un sistema de seguridad, aún estamos lejos de alcanzar los objetivos propuestos (2012: 16).

La Procuraduría General de la República, en su informe denominado Los feminicidios en la República Dominicana (Resumen 2005-2011) da a conocer que los feminicidios íntimos —asesinatos de mujeres perpetrados por hombres con los que las víctimas tenían o habían tenido una relación íntima, familiar, de convivencia o afín a estafueron: 98 en 2005, 99 en 2006, 89 en 2007, 131 en 2008, 92 en 2009, 97 en 2010, y 127 en 2011.

Según el Boletín mensual de la Oficina Nacional de Estadísticas, Feminicidio intimo en República Dominicana, las mujeres que en el año 2008 murieron por feminicidio íntimo fueron en su mayoría mujeres jóvenes con edad entre 20 y 29 años (35\%); y la zona con mayor número de muertes fue la metropolitana (2009: 2).

La violencia de género en la República Dominicana constituye un grave problema de salud pública. Según el tercer informe sobre violencia de género del Centro Reina Sofía, de España, nuestro país ocupa el primer lugar en cuanto a la violencia contra la mujer en América Latina. En comparación con el resto de la región y Estados Unidos, las mujeres asesinadas en el entorno doméstico son más del doble de la media, y cinco veces más que la media de Europa (Córdoba y Pérez, 2012: 6-7). Según el informe Feminicidio en la República Dominicana, el 70\% de los feminicidios cometidos durante el período estudiado (enero-diciembre del año 2001) fueron íntimos (Pola, 2002: 64).

La violencia de género ha sido explicada como resultado de factores sociales, culturales y políticos (Ramos, 1991) o como consecuencia de un aprendizaje familiar y social (Rodríguez, 2000). Heise destaca la naturaleza multifacética del fenómeno y propone un modelo ecológico (1998). 
Güezmes (2004) recurre al modelo ecológico propuesto por Heise y explica la violencia de género considerando:

a) Factores del individuo - haber presenciado episodios de violencia entre sus padres, ser propietario de armas, el abuso de alcohol y otras sustancias, la pérdida del estatus socioeconómico y la asociación con delincuentes-.

b) Relaciones cercanas - la dominación masculina en la familia, el control masculino de bienes, los conflictos conyugales y el aislamiento de la mujer en la familia-.

c) Contextos comunitarios - bajo estatus de la mujer, la falta de servicios de apoyo, altos niveles de desempleo y delincuencia, el aislamiento de la mujer y la presencia de violencia en la comunidad-.

d) Factores relativos a la estructura de la sociedad -la existencia de desigualdades importantes entre hombres y mujeres, la rigidez de los roles de los géneros, las normas culturales que respaldan el derecho del hombre a mantener relaciones sexuales extramaritales, la aceptación social del castigo físico, la violencia interpersonal y la percepción de que la mujer es propiedad del hombre-

En la República Dominicana, se han realizado varias encuestas en el territorio nacional en las que se investiga la violencia contra la mujer: la Encuesta experimental demográfica y de salud (1999); la Encuesta demográfica y de salud 2002 (Endesa, 2002); y la Encuesta demográfica y de salud 2007 (Endesa, 2007).

En la encuesta Endesa (2002), se entrevistaron a 23,384 mujeres, con edad entre 15 y 49 años; y a 2,833 hombres con edad entre 15 y 59 años. En el cuestionario aplicado a la población femenina, un módulo estuvo destinado a indagar la violencia contra la mujer. De igual modo, en el cuestionario aplicado a los hombres, un módulo estuvo destinado a indagar acerca de las actitudes del hombre hacia la mujer. 
Los resultados de la Endesa (2002) más relevantes para el tema que nos ocupa aparecen resumidos a continuación. También se citan otras fuentes, como, por ejemplo, la Endesa (2007).

\subsection{LAS VÍCTIMAS}

Según los datos suministrados por la Oficina Nacional de Estadísticas, la violencia conyugal se incrementó entre 2004 y 2008, en un $2.2 \%$, entre las mujeres con edad entre 15 y 49 años. El porcentaje de mujeres víctimas de alguna forma de violencia pasó de un 27.6\% a un 29.8\% (Boletín Mensual, 2009).

La violencia intrafamiliar contra la mujer, según la Endesa 2002, es mayor en la zona urbana (24.8\%) que en la zona rural (21.9\%). Entre las mujeres más jóvenes, con edad entre los 15 y 24 años, un $66.2 \%$ ha sido objeto de algún tipo de violencia conyugal. Este tipo de violencia se reduce a medida que aumenta la edad de la mujer: $28 \%$ entre las que tienen entre 25 y 34 años, y un $21 \%$ entre las que tienen entre 45 y 49 años.

Según la edad de la mujer, la violencia conyugal varía entre las que han estado casadas o unidas consensualmente: $27 \%$ de las que tienen entre 15 y 34 años ha sido víctima de la violencia conyugal; $23 \%$ entre las de 35-44 años y 16\% entre las de 45-49 años. Entre las mujeres sin marido o sin compañero, ha padecido violencia conyugal en el 48\% entre los 15-24 años, el 40\% entre 25-44 años y el 32\% entre $45-49$ años.

Cuando la unión es consensual, la violencia conyugal es mayor $(28.3 \%)$ que cuando la mujer está casada (15.3\%). Es mayor cuando la mujer ha emigrado al lugar donde reside $(26.7 \%)$ que cuando es nativa de la región (21.4\%); y disminuye a medida que aumenta la escolaridad de la mujer: el $28 \%$ de las maltratadas no completó la enseñanza primaria y solo el $17.6 \%$ de las que llegaron a la universidad fueron víctimas de la violencia conyugal. 
La actividad laboral de las mujeres se asocia con la violencia conyugal. El 19.4\% de mujeres víctimas de violencia realiza un trabajo de cuello blanco; el 33.1\% trabaja en casas de familia; el $31.9 \%$ realiza labores manuales; el 33.6\% trabaja en otro tipo de servicio; y un $24 \%$ de estas mujeres son empleadas en el área comercial. Cuando la mujer no trabaja fuera del hogar, la violencia se reduce $(21 \%)$. Eso por un lado, por otro lado, las mujeres víctimas de la violencia conyugal acusan al marido (52.3\%) o al exmarido (10.4\%), lo que significa que el 63\% de ellas ha sido agredida por la pareja actual o anterior. En sentido general, la mayor evidencia de violencia ocurre cuando termina la relación (40.1\%).

Según la Oficina Nacional de Estadísticas, el mayor porcentaje de víctimas de la violencia intrafamiliar se encuentra entre las mujeres separadas, viudas o divorciadas (37.4\%). Aproximadamente una tercera parte de las mujeres no se considera en igualdad de derechos con el hombre, y un $9.1 \%$ justifica que el hombre le pegue a su esposa. Con respecto a esos indicadores, tenemos: $53.8 \%$ y $21.5 \%$ en el Suroeste; $42.9 \%$ y $12.6 \%$ en la zona rural; $30.3 \%$ y $4.6 \%$ en el Distrito Nacional y en la Región Este del país (Boletín Mensual, 2009).

A medida que aumenta el nivel de ingresos de la mujer y su estatus ocupacional, mejor es la percepción que ella tiene de sus derechos y menos justifica que el hombre le pegue. Lo mismo ocurre cuando la escolaridad y el acceso a la información aumentan.

Las mujeres más jóvenes tienden a ser más conservadoras. Entre las que tienen entre 15 y 19 años, el $44.1 \%$ no se considera en igualdad de derechos con el hombre; y entre las que tienen entre 45 y 49 años de edad, un 33.5\%. El 12.7\% de las adolescentes justifica que el marido le pegue a la esposa o compañera; y entre aquellas que tienen entre 20 y 44 años, solo un $8 \%$ lo justifica.

El 46.7\% de mujeres en unión consensual se percibe con menos derechos que el hombre; y el $12.2 \%$ de ellas justifica que el hombre le pegue a su pareja. Entre las casadas, un $28.4 \%$ tiene la misma 
percepción que las del primer grupo, y un $4.2 \%$ se une al segundo. Las mujeres adultas jóvenes, con edad entre 20 y 34 años, padecen con más frecuencia la violencia física y el maltrato emocional de parte de su pareja o expareja. Por el contrario, las mujeres entre 45 y 49 años son menos proclives a ser maltratadas.

Según la Endesa 2007, una gran proporción de las mujeres que han vivido situaciones de violencia tienen antecedentes de ese perjuicio en su hogar de procedencia, lo que sugiere que se trata de patrones de conducta percibidos como normales. Asimismo, la Endesa 2007 indica que el porcentaje de mujeres que ha experimentado alguna forma de violencia conyugal es menor cuando estas participan en cuatro o más decisiones dentro del hogar (24\%) que cuando participan en tres o menos decisiones $(34 \%)$. Por otra parte, un mayor número de mujeres son víctimas de violencia cuando tienen cónyuges que ejercen control sobre sus vidas $(77 \%)$, en comparación con las que tienen cónyuges que no ejercen ese control (14\%).

\subsection{LOS AGRESORES}

Según la Endesa 2002, el 33.5\% de los hombres considera que el cónyuge tiene la última palabra con respecto a gastos mayores y a las visitas que pueda realizar su compañera. El 66.2\% cree que, si no es económicamente necesario, la mujer no debe trabajar fuera del hogar. Un $47.7 \%$ se considera con derecho a sancionar a su pareja si se niega a tener relaciones sexuales; y un 11\% justifica pegar a la compañera por cualquier motivo. Aunque un porcentaje mayor de campesinos justifica pegar a su pareja, la violencia conyugal de tipo físico es mayor entre los hombres que residen en la ciudad.

Si bien es cierto que a mayor nivel educativo del hombre, menor es su justificación de la violencia conyugal y mayor su aceptación de que la mujer trabaje fuera del hogar, no menos cierto es que un $41 \%$ de los hombres universitarios no acepta que la mujer trabaje fuera del entorno doméstico. Un dato importante es que entre los 
hombres más jóvenes, con edad entre 15 y 34 años, se encuentra el mayor porcentaje de los que se creen que el hombre tiene la última palabra. Además, los hombres más jóvenes son más proclives a la violencia conyugal.

Según datos suministrados por Luis Vergés, director del Centro de Intervención Conductual para Hombres (República Dominicana), la mayoría de los hombres que maltratan a sus parejas no son analfabetos, no tienen trastornos psiquiátricos (95\%); no están desempleados ni son drogadictos. Solo el $2 \%$ de los hombres que llegan al centro son analfabetos. Un 33\% tiene estudios superiores y de postgrado; un $40 \%$ tiene educación media y un $25 \%$ tiene educación básica. El 86\% de los hombres agresores tiene al menos un empleo; el $12 \%$ es desempleado, y el $1 \%$ es pensionado. El $62 \%$ de los hombres que llegan al Centro no reporta abuso de alcohol; el 38\% sí lo hace y el 6\% reporta uso de drogas. Sin embargo, un 52\% de los usuarios del Centro reveló que recibió maltrato durante su infancia. Del total, el $42 \%$ son solteros, un $31 \%$ son casados, el $20 \%$ está en unión libre, el $6 \%$ está separado y el 1\% está divorciado. El 36\% que ha asistido en los últimos dos años, tienen edad entre los 33 y los 40 años; el 29\%, de 40 a 50 años; el 21\%, de 20 a 30 años; y el 13\%, más de 50 años (Listín Diario, 15/11/2010: 1 y 8A).

La Organización Panamericana de la Salud (OPS, 1998) planteó la necesidad de que las investigaciones sobre la violencia conyugal contemplen la figura del agresor para conocer la etiología del problema y desarrollar intervenciones científicamente sustentables con fines de prevención y de rehabilitación. Sin embargo, algunos autores opinan que es imposible curar al hombre agresor porque no experimenta sentimiento de culpa y porque responde de un modo egosintónico a comportamientos aprendidos desde la infancia. A juicio de Barea, el trabajo con los agresores carece de sentido y el esfuerzo debe concentrarse en la protección y tratamiento de las víctimas (2004: 101). 
Montás y Herrera afirman que, además de la prevención y protección de las víctimas por parte del sistema de justicia, los agresores que sigan insertados en la vida productiva reclutarán nuevas víctimas, de conformidad con una dinámica cíclica de dominio y posesión dentro de la familia (2011: 397). Por esa razón, realizaron una investigación con el fin de ampliar los conocimientos sobre los hombres agresores. Se utilizó una muestra de 34 hombres agresores y se enfatizó la necesidad de desarrollar estrategias preventivas y/o de tratamiento del hombre agresor (2011: 396).

Los resultados del estudio de Montás y Herrera sugieren que los hombres agresores en el contexto familiar tienen una inteligencia no verbal promedio, independientemente de su edad o nivel educativo. Pero se constata una disfunción en el orden de la categorización/abstracción, la flexibilidad cognitiva, la toma de decisiones y la capacidad para resistir la perseveración. Los errores de perseveración demuestran que hay dificultad para ser cognitivamente flexibles y capaces de adaptarse a una situación dada (2011: 417-418).

\section{Abuso infantil y violencia conyugal}

\subsection{ESTUDIOS SOBRE LA ASOCIACIÓN ENTRE LA VIOLENCIA CONYUGAL Y EL ABUSO INFANTIL}

Estudios realizados (Edleson, 1999; Appel y Holden, 2003) han encontrado que existe una relación entre la violencia conyugal y el abuso infantil. La madre victimizada es, con frecuencia, incapaz de proteger a sus hijos del abuso. Víctima de la violencia, ella a menudo está en condiciones que le dificultan el ejercicio de su rol como madre o le impiden satisfacer las necesidades de sus hijos. Además, las madres maltratadas tienen más probabilidad de ser agresivas con sus hijos, debido a los niveles de estrés y depresión que experimentan como consecuencia de la violencia que han padecido. En ese sentido, conviene tener en cuenta que la violencia 
física puede desplazarse hacia los hijos, así como también la violencia psicológica. La negligencia física o emocional de la madre hacia los hijos también puede ser una consecuencia de la violencia conyugal.

Por exposición a un ambiente familiar violento, los hijos aprenden a comportarse de un modo violento identificándose con el agresor. Los niños descubren que la violencia es una forma de ejercer el poder y una estrategia para enfrentar determinadas situaciones. Las niñas, por su parte, aprenden a adoptar conductas de sumisión y de obediencia, como una reacción defensiva para evitar el maltrato.

Cuando el padre agrede a la madre, el conflicto de lealtades y la confusión de los roles alteran las condiciones familiares para el desarrollo; y afectan el sentimiento de seguridad personal del hijo, así como su confianza en los demás, lo que trastorna el proceso de internalización de las normas. La violencia conyugal afecta a los hijos, quienes tienen más probabilidad de desarrollar conductas agresivas y antisociales, así como ansiedad, tendencia a la inhibición, baja autoestima y sentimiento de culpa (Fantuzzo et al., 1991: 258-265).

También se observan otras consecuencias como menor competencia social, bajo rendimiento académico y estrés postraumático (Adamson y Thompson, 1998: 213-232; Maker et al., 1998: 574-589). A largo plazo, la exposición continuada del niño a episodios de violencia doméstica y la aplicación de sistemas de castigos basados en el ejercicio del poder constituyen predictores del riesgo de que el sujeto ejerza violencia contra su pareja en el futuro. 


\subsection{El abuso infantil en la REPÚbliCA DOMINICANA}

Desde el punto de vista legal, en nuestro país se considera "abuso infantil"1 toda conducta que provoque o pueda provocar - por acción u omisión- daño a la integridad o salud física, psicológica o sexual de un menor de edad por parte de cualquier persona, progenitor o pariente, educador o persona a cargo de su cuidado (Ley N. ${ }^{\circ}$ 24-97, Art. 351-2).

Según UNICEF, en la República Dominicana un 9.4\% de los padres considera que el castigo es necesario para educar a los hijos apropiadamente; un 56.7\% usa el castigo físico moderado; un 3.5\% usa el castigo físico severo; y un $71.2 \%$ de los niños ha padecido castigo psicológico, como regaño verbal e insulto. El 83.4\% de los niños de 2 a 14 años ha experimentado alguna forma de castigo físico o psicológico; y el $87.4 \%$ de los niños más pobres padecen castigo, mientras solo el $77.8 \%$ de los más ricos son víctimas de castigo (2012: 13-32).

\subsection{INVESTIGACIÓN CLÍNICA}

La investigación que presentamos a continuación sobre la violencia conyugal en la República Dominicana es un estudio clínico, una exploración psicológica situada en el campo de la salud mental.

El problema que se plantea es de la posible relación entre dos variables: el maltrato de la pareja y el abuso infantil, en la familia dominicana. Con el propósito de desarrollar y resolver el problema, se evaluó un grupo de 90 pacientes masculinos y adultos con respecto a las variables estudiadas en el marco de una psicoterapia psicodinámica. El estudio se realizó con pacientes de la consulta privada residentes en Santo Domingo, pertenecientes a todas las

1 Sería más preciso hablar de abuso infanto-juvenil. No obstante, emplearemos el término de uso más frecuente. 
clases sociales y con niveles educativos diferentes - profesionales universitarios, técnicos y obreros calificados-, que fueron sometidos a una psicoterapia breve de orientación analítica.

La pregunta de la que parte muestra investigación es la siguiente: $¿$ Es el abuso de que fueron objeto los hombres durante su infancia un factor determinante del maltrato que ellos han ejercido o ejercen sobre sus parejas? Esta interrogante nos condujo a la siguiente hipótesis: existe una asociación significativa entre el abuso infantil y el maltrato de la pareja durante la adultez. Con el propósito de comprobar la hipótesis, se hizo un estudio comparativo con un diseño factorial $(2 \times 2)$.

En nuestra investigación, la variable independiente es el abuso infantil. Después de que se exploró la conducta de los pacientes, se utilizó una escala ordinal con rangos de 0 a 5 , para evaluar a cada sujeto de la muestra con respecto a esta variable. Luego, con los puntajes de esa escala, y a partir de la mediana (1), se dicotomizó la variable; y se dividieron los sujetos en dos grupos. Aquellos que estaban por encima de la mediana conformaron el grupo de alto abuso infantil (A. I.); y los que estaban en la mediana, o por debajo de ella, conformaron el grupo de bajo abuso infantil.

El maltrato de la pareja (M. P.), como variable dependiente, fue definido operacionalmente como el puntaje obtenido en una escala ordinal con rangos de 1 a 3 . Con los puntajes de esa escala, y a partir de la mediana (1), se dicotomizó la variable; y se dividieron los sujetos en dos grupos. Aquellos que estaban por encima de la mediana conformaron el grupo de alto maltrato de la pareja (M. P.); y los que estaban en la mediana o por debajo de ella conformaron el grupo de bajo maltrato de la pareja.

Con el propósito de establecer la asociación estadística entre el abuso infantil (A. I.) y el maltrato de la pareja (M. P.), utilizamos el chi cuadrado $\left(\mathrm{X}^{2}\right)$. Los resultados de la investigación, que aparecen representados a continuación, confirman la hipótesis. 
Tabla N. ${ }^{\circ} 1$

Resultados sobre el maltrato de la pareja y el abuso infantil

\begin{tabular}{|c|c|c|c|c|c|}
\hline Paciente & $\begin{array}{l}\text { Maltrato de } \\
\text { la pareja }\end{array}$ & Edad & $\begin{array}{c}\text { Nivel } \\
\text { educativo }\end{array}$ & $\begin{array}{c}\text { Estatus } \\
\text { social }\end{array}$ & $\begin{array}{l}\text { Abuso } \\
\text { infantil }\end{array}$ \\
\hline 1. & 2 & 28 & OC & B & 2 \\
\hline 2. & 3 & 32 & OC & B & 2 \\
\hline 3. & 1 & 28 & $\mathrm{PU}$ & $\mathrm{M}$ & 2 \\
\hline 4. & 1 & 53 & $\mathrm{PU}$ & $\mathrm{M}$ & 0 \\
\hline 5. & 1 & 45 & OC & $\mathrm{B}$ & 1 \\
\hline 6. & 1 & 26 & $\mathrm{~T}$ & $\mathrm{M}$ & 1 \\
\hline 7. & 1 & 51 & $\mathrm{PU}$ & $\mathrm{A}$ & 4 \\
\hline 8. & 1 & 37 & $\mathrm{PU}$ & $\mathrm{M}$ & 1 \\
\hline 9. & 1 & 25 & $\mathrm{PU}$ & A & 2 \\
\hline 10. & 1 & 40 & OC & B & 0 \\
\hline 11. & 1 & 28 & $\mathrm{PU}$ & A & 0 \\
\hline 12. & 1 & 31 & $\mathrm{PU}$ & A & 0 \\
\hline 13. & 1 & 40 & OC & $\mathrm{B}$ & 2 \\
\hline 14. & 1 & 30 & OC & $\mathrm{B}$ & 1 \\
\hline 15. & 1 & 38 & $\mathrm{PU}$ & A & 0 \\
\hline 16. & 2 & 27 & OC & $\mathrm{B}$ & 3 \\
\hline 17. & 1 & 28 & $\mathrm{~T}$ & $\mathrm{M}$ & 0 \\
\hline 18. & 1 & 34 & $\mathrm{~T}$ & $\mathrm{M}$ & 1 \\
\hline 19. & 1 & 29 & $\mathrm{~T}$ & $\mathrm{M}$ & 1 \\
\hline 20. & 1 & 36 & OC & $\mathrm{B}$ & 2 \\
\hline 21. & 1 & 25 & $\mathrm{~T}$ & M & 0 \\
\hline 22. & 1 & 53 & $\mathrm{~T}$ & $\mathrm{M}$ & 1 \\
\hline 23. & 1 & 60 & $\mathrm{~T}$ & $\mathrm{M}$ & 0 \\
\hline 24. & 2 & 65 & $\mathrm{~T}$ & $\mathrm{M}$ & 2 \\
\hline 25. & 1 & 30 & $\mathrm{PU}$ & $\mathrm{A}$ & 2 \\
\hline 26. & 2 & 26 & OC & B & 3 \\
\hline 27. & 1 & 28 & $\mathrm{~T}$ & M & 2 \\
\hline 28. & 1 & 31 & $\mathrm{~T}$ & $\mathrm{M}$ & 0 \\
\hline 29. & 1 & 34 & $\mathrm{~T}$ & $\mathrm{M}$ & 0 \\
\hline
\end{tabular}




\begin{tabular}{|c|c|c|c|c|c|}
\hline Paciente & $\begin{array}{l}\text { Maltrato de } \\
\text { la pareja }\end{array}$ & Edad & $\begin{array}{c}\text { Nivel } \\
\text { educativo }\end{array}$ & $\begin{array}{c}\text { Estatus } \\
\text { social }\end{array}$ & $\begin{array}{l}\text { Abuso } \\
\text { infantil }\end{array}$ \\
\hline 30. & 1 & 53 & OC & B & 0 \\
\hline 31. & 2 & 27 & OC & B & 4 \\
\hline 32. & 1 & 35 & $\mathrm{~T}$ & $\mathrm{M}$ & 0 \\
\hline 33. & 2 & 26 & $\mathrm{PU}$ & $\mathrm{A}$ & 2 \\
\hline 34. & 1 & 31 & OC & $\mathrm{A}$ & 1 \\
\hline 35. & 1 & 28 & $\mathrm{PU}$ & $\mathrm{B}$ & 0 \\
\hline 36. & 1 & 59 & $\mathrm{PU}$ & $\mathrm{A}$ & 4 \\
\hline 37. & 1 & 28 & $\mathrm{PU}$ & M & 0 \\
\hline 38. & 1 & 65 & $\mathrm{PU}$ & $\mathrm{M}$ & 0 \\
\hline 39. & 3 & 26 & OC & $\mathrm{B}$ & 2 \\
\hline 40. & 2 & 29 & OC & $\mathrm{B}$ & 2 \\
\hline 41. & 1 & 60 & $\mathrm{PU}$ & $\mathrm{M}$ & 1 \\
\hline 42. & 1 & 28 & OC & $\mathrm{B}$ & 0 \\
\hline 43. & 1 & 39 & $\mathrm{PU}$ & $\mathrm{M}$ & 1 \\
\hline 44. & 1 & 62 & $\mathrm{PU}$ & $\mathrm{A}$ & 0 \\
\hline 45. & 1 & 54 & OC & B & 0 \\
\hline 46. & 1 & 41 & $\mathrm{PU}$ & $\mathrm{M}$ & 1 \\
\hline 47. & 1 & 42 & $\mathrm{PU}$ & $\mathrm{M}$ & 1 \\
\hline 48. & 1 & 31 & $\mathrm{PU}$ & $\mathrm{A}$ & 0 \\
\hline 49. & 2 & 45 & $\mathrm{PU}$ & $\mathrm{A}$ & 5 \\
\hline 50. & 2 & 35 & OC & B & 0 \\
\hline 51. & 1 & 50 & $\mathrm{PU}$ & $\mathrm{A}$ & 0 \\
\hline 52. & 2 & 45 & OC & $\mathrm{B}$ & 5 \\
\hline 53. & 1 & 31 & OC & B & 1 \\
\hline 54. & 2 & 35 & OC & $\mathrm{B}$ & 3 \\
\hline 55. & 2 & 42 & $\mathrm{PU}$ & $\mathrm{M}$ & 2 \\
\hline 56. & 1 & 36 & $\mathrm{PU}$ & A & 0 \\
\hline 57. & 1 & 48 & OC & B & 0 \\
\hline 58. & 1 & 45 & $\mathrm{PU}$ & $\mathrm{A}$ & 0 \\
\hline 59. & 1 & 50 & OC & $\mathrm{B}$ & 1 \\
\hline 60. & 2 & 33 & $\mathrm{PU}$ & $\mathrm{M}$ & 0 \\
\hline
\end{tabular}




\begin{tabular}{|c|c|c|c|c|c|}
\hline Paciente & $\begin{array}{c}\text { Maltrato de } \\
\text { la pareja }\end{array}$ & Edad & $\begin{array}{c}\text { Nivel } \\
\text { educativo }\end{array}$ & $\begin{array}{c}\text { Estatus } \\
\text { social }\end{array}$ & $\begin{array}{l}\text { Abuso } \\
\text { infantil }\end{array}$ \\
\hline 61. & 1 & 43 & PU & A & 1 \\
\hline 62. & 1 & 50 & $\mathrm{PU}$ & $A$ & 0 \\
\hline 63. & 2 & 48 & $\mathrm{PU}$ & A & 1 \\
\hline 64. & 1 & 32 & $\mathrm{PU}$ & A & 0 \\
\hline 65. & 1 & 36 & $\mathrm{PU}$ & $\mathrm{M}$ & 0 \\
\hline 66. & 2 & 44 & $\mathrm{OC}$ & B & 2 \\
\hline 67. & 1 & 41 & $\mathrm{PU}$ & A & 1 \\
\hline 68. & 2 & 48 & $\mathrm{PU}$ & $\mathrm{M}$ & 0 \\
\hline 69. & 1 & 42 & $\mathrm{OC}$ & B & 1 \\
\hline 70. & 1 & 45 & $\mathrm{PU}$ & $\mathrm{A}$ & 1 \\
\hline 71. & 2 & 50 & $\mathrm{PU}$ & A & 0 \\
\hline 72. & 1 & 46 & $\mathrm{PU}$ & A & 1 \\
\hline 73. & 2 & 43 & $\mathrm{PU}$ & $\mathrm{A}$ & 0 \\
\hline 74. & 2 & 47 & $\mathrm{PU}$ & A & 1 \\
\hline 75. & 2 & 41 & $\mathrm{OC}$ & $\mathrm{B}$ & 1 \\
\hline 76. & 1 & 45 & $\mathrm{PU}$ & A & 0 \\
\hline 77. & 1 & 47 & $\mathrm{OC}$ & $\mathrm{B}$ & 1 \\
\hline 78. & 2 & 47 & $\mathrm{PU}$ & $\mathrm{M}$ & 0 \\
\hline 79. & 2 & 43 & $\mathrm{PU}$ & $\mathrm{M}$ & 1 \\
\hline 80. & 2 & 45 & $\mathrm{OC}$ & $\mathrm{B}$ & 0 \\
\hline 81. & 2 & 41 & $\mathrm{PU}$ & A & 0 \\
\hline 82. & 1 & 50 & $\mathrm{PU}$ & A & 1 \\
\hline 83. & 2 & 30 & $\mathrm{OC}$ & $\mathrm{B}$ & 2 \\
\hline 84. & 2 & 33 & $\mathrm{PU}$ & $\mathrm{M}$ & 3 \\
\hline 85. & 2 & 42 & $\mathrm{OC}$ & $\mathrm{B}$ & 2 \\
\hline 86. & 2 & 54 & $\mathrm{PU}$ & A & 3 \\
\hline 87. & 2 & 29 & $\mathrm{OC}$ & B & 5 \\
\hline 88. & 2 & 61 & $\mathrm{PU}$ & $\mathrm{M}$ & 3 \\
\hline 89. & 2 & 47 & $\mathrm{PU}$ & $\mathrm{A}$ & 2 \\
\hline 90. & 2 & 53 & $\mathrm{PU}$ & $\mathrm{A}$ & 5 \\
\hline
\end{tabular}


Tabla N. ${ }^{\circ} 2$

Asociación entre el maltrato de la pareja y el abuso infantil

\begin{tabular}{|c|c|c|c|}
\hline $\begin{array}{c}\text { Maltrato } \\
\text { de la pareja }\end{array}$ & Alto A. I. & Bajo A. I. & Total \\
\hline- & 08 & 48 & 56 \\
\hline+ & 22 & 12 & 34 \\
\hline Total & 30 & 60 & 90 \\
\hline
\end{tabular}

\begin{tabular}{|c|c|c|c|c|}
\hline & $\mathbf{X} 2$ & $\mathbf{P}$ & Significatividad & $\begin{array}{c}\text { Probabilidad } \\
\text { de error }\end{array}$ \\
\hline $\mathbf{X}^{\mathbf{2}}$ & 24.202 & 0.000 & Altísima & $1 / 1000$ o menos \\
\hline Corregida & 24.070 & 0.000 & Altísima & $1 / 1000$ o menos \\
\hline GL & 1 & & & \\
\hline
\end{tabular}

\section{Interpretación etnopsicoanalítica}

\subsection{LA MATRIFOCALIDAD EN LA FAMILIA DOMINICANA}

La estructura de la familia dominicana, es decir, el sistema de relaciones de parentesco que asocia y organiza los personajes del grupo doméstico, es esencialmente matrifocal. El término "matrifocal", original de la etnología afroamericana, significa que las relaciones entre los miembros de la familia se focalizan más en la díada madre-hijo que en la relación de alianza.

Aunque la explicación histórica de la familia matrifocal del Caribe reenvíe a la esclavitud y a la economía de la plantación, este enfoque no permite comprender cómo ese tipo de familia pudo sobrevivir a la situación que le dio origen. La resistencia al cambio de la familia matrifocal obliga a trascender un análisis puramente determinista. 
La alianza no constituye en la República Dominicana el principio fundamental del dispositivo familiar. Por el contrario es siempre incierta y aleatoria, frágil y efímera. La madre es el punto focal, el lugar de convergencia y de redifusión de las relaciones familiares. Ella está en la base de los vínculos que se establecen entre los demás personajes de la familia. Pero, más que un personaje, ella es la representante de un linaje que conduce hasta la abuela materna.

Sin embargo, para posibilitar la relación entre los miembros del grupo familiar y evitar su reabsorción en la figura materna, es necesario preservar una distancia mínima con respecto a la madre, por lo que resulta necesario tener en cuenta el conjunto de las relaciones entre los miembros de la familia y su covarianza. La familia matrifocal no es la supervivencia de una colección de elementos del pasado, sino un sistema significante con su lógica propia. Por esa razón, su estudio no puede limitarse a observar y medir actitudes y conductas. Para descubrir la significación psicológica de estas, es necesario reenviarlas a un código que las sitúe con respecto a un sistema de significaciones. Desde el punto de vista psíquico, la matrifocalidad designa un dispositivo libidinal en el que el deseo de la madre es fundamental. Es imprescindible atender a la dimensión del deseo que circula entre los personajes de la familia.

Una condición necesaria para la existencia del dispositivo matrifocal es que el vínculo madre-hijo nunca se rompa. Los hijos se quedan con la madre durante la infancia, y vuelven donde ella durante la adultez. En ese sentido, la casa materna constituye un verdadero espacio transicional por el que nunca se deja de transitar.

Si la separación de los hijos con respecto a la madre biológica es para los niños una situación difícil, la sociedad dispone, para esa ocasión, de la práctica de la adopción, la cual suele ocurrir estando la madre viva y en condiciones de criar. Aunque el hijo tenga una madre biológica, existen otras madres sustitutivas en una proximidad 
metonímica con respecto a la progenitora: una hermana mayor, una tía, una abuela. En realidad, deberíamos hablar de un polo materno extenso.

En la familia matrifocal dominicana, el padre siempre ocupa una posición periférica o relativamente periférica. Aun cuando se trate de un hombre casado, autoritario y controlador, que les ha dado el apellido a sus hijos, nunca es un verdadero patriarca, un paterfamilias. Aunque esté presente en el hogar y tenga la pretensión de dirigir el grupo doméstico, las reglas de conducta que él impone a los demás miembros de la familia están poco interiorizadas en él mismo. Además, el padre dominicano es frecuentemente denigrado por la madre. Por esas razones, la figura paterna no suele ser el soporte de una función simbólica cuyo reconocimiento, por parte del hijo, garantice la función paterna.

Lacan prosiguió la empresa freudiana de revalorización de la función paterna, al erigir el concepto de Nombre-del-Padre en significante de esta y al hacer de la familia el crisol de la norma. Sin embargo, la nominación paterna, según Lacan, no es la simple transmisión del patronímico, sino todo un reconocimiento simbólico. Por esa razón, de acuerdo con Roudinesco, el concepto de Nombre-del-Padre no queda invalidado por el hecho de que el hijo pueda heredar el patronímico o apellido de su madre y no el de su padre (2006: 207).

Cuando el padre está ausente y la familia está compuesta por la mujer, sus hijos y el concubino, este último no es verdaderamente un padrastro de los hijos. Y, aunque sea violento, no ejercerá una verdadera autoridad ni desempeñará una función paterna auténtica. En ese sentido, es necesario distinguir el verdadero castigo de una simple demostración de fuerza. El castigo implica una legitimación de la autoridad, lo que exige a su vez, de parte del interdictor, la interiorización de las normas exigidas a los demás. En la familia matrifocal, la frecuencia del acting out agresivo se explica por un diferimiento de la castración simbólica, determinado por la falta 
de reconocimiento simbólico de la función paterna. $Y$ es el yo ideal -no el superyó- el que orienta la conducta. La tiranía del hombre no basta para fundar en el seno de la familia un verdadero orden patriarcal. Su mandato solo es analizable en los términos del dispositivo matrifocal.

El padre, en la familia dominicana, no es el garante de la ley ni asegura la transmisión del nombre. Que un hijo no lleve el apellido de su padre es un hecho frecuente; y la ilegitimidad no reviste el carácter dramático que tiene en las sociedades patriarcales. En ese sentido, la prevalencia del linaje uterino conduce a la denegación de la alianza, y apuntala la fuerza endogámica de un tipo de familia cuya fuente de energía es esencialmente materna.

El padre dominicano no solo está relativamente ausente. Es necesario reconocer también que la madre lo mantiene alejado. Y, cuando él se distancia completamente, ella no lo sustituye por otra figura paterna como podría ser el padre de ella o su hermano mayor. Incluso cuando la madre se eclipsa discretamente detrás de su compañero, no tarda en denigrarlo o en denunciar su inconsistencia, lo que resalta su propia constancia y revela la recompensa que ella espera: la fidelidad de los hijos. De ese modo, se crea un equilibrio que el padre siempre amenaza con perturbar, cuando él pretende ocupar esa posición inocupable definida como la de aquel por quien el conflicto se hace regularmente presente. En el discurso materno, la cuestión del padre siempre se plantea con un valor negativo.

La familia matrifocal dominicana no es, sin embargo, una desviación provisional de la familia nuclear occidental. Su persistencia a través del tiempo revela que cuenta con una economía libidinal, en la que el vínculo madre-hijo constituye la articulación esencial de esa organización original de las relaciones de parentesco. 
Según M. J. Pola, solo el 36.7\% de los hogares urbanos de la República Dominicana responde al modelo de la familia nuclear biparental con hijos (2006: 27). Pero, incluso en estos hogares, si escuchamos el discurso de los hombres de la clase media y alta que componen parte de la muestra de la investigación, no tardamos en descubrir una "matrifocalidad atenuada" o una "tendencia a la matrifocalidad".

En la clase media y alta del medio urbano de Santo Domingo, en las que el ideal es la relación conyugal, la necesidad económica o las exigencias femeninas con sus anhelos de proyección social determinan que la mujer trabaje fuera del hogar, aun cuando "mantenerla" 2 por lo menos parcialmente, confiera al hombre seguridad y control.

Sin embargo es necesario reconocer que en la clase media y alta la independencia económica de la mujer afecta negativamente el narcisismo masculino. En la clase baja marginal, aun cuando ella trabaje fuera del hogar, el hombre completa sus ingresos para su manutención. Pero, cuando - como suele suceder en la clase media y alta- ella se mantiene o gana más que el compañero esposo, o cuando él cree que pierde el control sobre ella, él deviene rígido, evoca la moral, defiende sus buenas intenciones y, en el mejor de los casos, recurre al psicólogo o al psiquiatra.

En la sociedad dominicana, la mirada del otro afecta el equilibrio narcisista. Importa considerablemente la opinión que circule en el vecindario, barrio o medio social. $\mathrm{Y}$, aunque el crimen pasional se asocie con la persecución y provoque vergüenza, el sentimiento de culpa no tiene un lugar preponderante.

2 Término ambiguo que significa tanto la obligación del proveedor económico como su anhelo de retenerla consigo. 
En la República Dominicana, la violencia conyugal debe considerarse como un síntoma postraumático. Creemos que el incremento de esta y de los feminicidios íntimos se debe al aumento del odio reprimido hacia la madre y a la necesidad imperiosa - dentro de la economía libidinal matrifocal- de desplazar hacia la pareja esa agresividad reprimida.

Los factores determinantes del incremento del odio hacia la madre pueden asociarse con la emigración y con una sustitución materna cada vez más precaria afectivamente, y cada vez más motivada por intereses económicos, como la remesa procedente del extranjero. En ese sentido, podríamos sustentar la hipótesis de una restricción afectiva del polo materno lo que, junto a un inicio temprano de la escolaridad en el niño y al trabajo fuera del hogar por parte de la madre, contribuirían al incremento de la hostilidad hacia ella. Todo parece indicar que la emigración y la aculturación contribuyen a modificar el polo materno, y a promover el aumento del abuso infantil y de la violencia conyugal.

A nuestro juicio, los hombres jóvenes de la República Dominicana son más conservadores - como indica la Endesa 2002 y otras fuentes- porque el debilitamiento del polo materno, por las razones indicadas anteriormente, los ha afectado de una manera más contundente. Las mujeres jóvenes, por su parte, serían más conservadoras por las mismas razones sociales; pero la economía libidinal matrifocal promovería en ellas el aprendizaje de conductas de sumisión.

Si la matrifocalidad — en sentido estricto o atenuado-implica un incesto focal potencial (André, 1987), se debe al rol periférico del padre. Pero existe el tabú del incesto y una estructura familiar, con su manera peculiar de vivir y de enfermar, diferente tanto de la familia occidental como de la familia africana.

Ahora bien, en la medida en que la familia nuclear dominicana moderna, con una matrifocalidad autóctona atenuada, vive, como 
la familia occidental, un proceso de desorden (Roudinesco, 2006) que en Occidente conlleva la disminución del poder que tradicionalmente tenía el paterfamilias, ¿cómo podemos distinguir los efectos de ese desorden si comparamos la crisis actual de la familia nuclear occidental y la crisis de la familia nuclear dominicana? En la República Dominicana, hay una aculturación que afecta tanto la tradición como ciertos logros y aprendizajes modernos asociados con la influencia de la cultura occidental, en el orden psicológico, científico, religioso e institucional, entre otros. Sin embargo, el desorden - es decir, la crisis de la familia moderna dominicana- se ha canalizado a través de su tendencia a la matrifocalidad autóctona. Por esa razón, el incremento moderno de los hogares monoparentales ha afectado menos a la sociedad dominicana; y por otra parte, el mayor acceso a la información y el incremento de la escolaridad no han compensado el deterioro del polo materno. De ahí que los adultos jóvenes de ambos sexos sean más conservadores, y que recientemente se hayan incrementado los feminicidios íntimos. Por tanto, no debe sorprender que, entre los usuarios del Centro de Intervención Conductual para Hombres, el 57\% tenga entre 20 y 40 años, y el 33\% tenga estudios superiores y de postgrado.

La estructura familiar en la sociedad occidental actual está cada día más confiada al poder materno, y cada vez más evadida de la antigua autoridad patriarcal, lo que no significa que sea una familia matrifocal como la del Caribe. Despojado de los ornamentos de su antigua sacralidad, el matrimonio - en constante declinaciónse ha convertido en un modo de conyugalidad afectiva; $y$, a menudo, en Occidente, está precedido por un período de unión libre. Los hijos, concebidos cada vez más al margen de los lazos matrimoniales, asisten a la boda de sus padres, quienes con más frecuencia se divorcian. Las mujeres quedan muchas veces en una situación monoparental, y sufren más las consecuencias de rupturas que, a veces, ellas mismas propician. En Francia, para citar solo un ejemplo, el matrimonio está en constante declinación. En París 
hay más hogares individuales que familiares. Entre el año 1990 y el año 2000, se pasó de 1.2 millones de hogares monoparentales a 1.7 millones (Le Monde, 10/2/2001).

El principio mismo de la autoridad sobre el que se fundó la familia occidental está hoy en crisis; y las referencias patriarcales están en decadencia. Sin embargo, existen rasgos de la familia moderna dominicana que no encontramos en la familia moderna occidental, lo que se explica por la tendencia a la matrifocalidad propia de nuestro grupo doméstico.

\section{Conclusión}

Los estudios realizados en otros países indican que existe una asociación entre la violencia intrafamiliar contra la mujer y el abuso infantil que el menor vive en el interior de su familia, con las consecuencias patológicas que esa experiencia tiene sobre los futuros adultos.

La investigación que hemos realizado en la República Dominicana prueba que existe una asociación entre el abuso infantil y la violencia conyugal, es decir, que el sujeto de sexo masculino que fue abusado o expuesto a la violencia intrafamiliar en su infancia tiene una alta probabilidad de ejercer la violencia conyugal en la adultez.

Sin embargo, para descubrir los motivos de la violencia conyugal en la República Dominicana, es necesario interpretar el sentido de las conductas de agresión intrafamiliar en el marco de la dinámica propia de la familia matrifocal, lo que requiere de una perspectiva etnopsicoanalítica.

Si la variación del polo materno se explica por diferentes factores -aculturación, emigración, escolaridad temprana, trabajo fuera del hogar, entre otros-, el incremento del odio hacia la figura materna, que el dispositivo matrifocal reprime, se desplaza hacia la pareja y provoca el aumento de la violencia conyugal. 
La diferencia, con respecto a dicho incremento, entre la familia occidental y la familia dominicana, se explicaría por la matrifocalidad o la tendencia a la matrifocalidad. La estructura de la familia occidental no obliga en igual medida a que el odio hacia la madre sea desplazado hacia la pareja, como sí ocurre en la familia matrifocal dominicana.

\section{Referencias bibliográficas}

Adamson, J. L. \& Thompson, R. A. (1998). Coping with interparental verbal conflict by children exposed to spouse abuse and children from nonviolent homes. Journal of Family Violence, 13(3), 213-232.

André, J. (1987). L'incest focal dans la famille noire antillaise. Crimes, conflits, structures. Paris: Presses Universitaires de France.

Appel, A. E. \& Holden, G. W. (1998). The co-ocurrence of spouse and physical child abuse: A review and appraisal. Journal of family psychology, 12(4), 578-599.

Barea, C. (2004). Manual para mujeres maltratadas (que quieren dejar de serlo). Barcelona: Editorial Océano.

Cáceres, F. \& Estévez, G. (2004). Violencia conyugal en la República Dominicana: Hurgando tras sus raíces. Santo Domingo: Mediabyte.

Córdoba, M. \& Pérez, A. (2012). Roles para el hombre y la mujer en casos vinculados con la violencia de pareja en Santo Domingo, 2011. Cienciay Sociedad, 37(1), 5-42.

Edleson, J. L. (1999). Children's witnessing of adult violence in the United States. Journal of Interpersonal Violence, 14(8), 839-870.

Encuesta Experimental de Demografia y Salud (1999). Santo Domingo: Centro de Estudios Sociales y Demográficos. 
Encuesta Demográfica y de Salud 2002 (2002). Santo Domingo: Centro de Estudios Sociales y Demográficos.

Encuesta Demografia y de Salud 2007 (2007). Santo Domingo: Centro de Estudios Sociales y Demográficos.

Fantuzzo, J. W., De Paola, L. M., Lambert, L., Martino, T., Anderson, G. \& Sutton, S. (1991). Effects of interparental violence on the psychological adjustment and competencies of young children. Journal of Counsulting and Clinical Psychology, 59(2), 258-265.

Güezmes, A. (2004). La violencia contra la mujer como problema de salud pública, en Santo Domingo: Profamilia.

Heise, L. (1998). Violence against women. An integrated ecological framework. Violence Against Women, 4(3), 262-290.

Maker, A.H., Kemmelmeier, M. Y. Peterson, CH. (1998). Longterm psychological consequences in women of witnessing parental physical comflict and experiencing abuse in childhood. Journal of Interpersonal Violence, 13, 574-589.

Montás, G. \& Herrera, J. (2011). Perfil neurocognitivo de agresores masculinos en contextos familiares como un subtipo de la agresión generalizada (2da. Parte). Ciencia y Sociedad, 36(3), 395-422.

Oficina Nacional de Estadísticas (2009). Feminicidio íntimo en República Dominicana. Boletín mensual, N. ${ }^{\circ}$ 15, 1-2.

Pola, M. J. (2002). Feminicidio en la República Dominicana: Un estudio de los casos ocurridos en los distritos judiciales de Profamilia Santo Domingo y Santiago en el periodo enero-diciembre del 2001. Santo Domingo: Editora Corripio. 
Pola, M. J. (2006). Las dominicanas y la violencia. Una aproximación al sistema de atención desde la justicia. Santo Domingo: Editora Corripio.

Pola, S. (2008). Feminicidio en República Dominicana 2000-2006. San José, Costa Rica: Asociación Centro Feminista de Información y Acción.

Ramos, C. (1991). El género en perspectiva. De la dominación universal a la representación múltiple. México: Universidad Autónoma Metropolitana Iztapalapa.

República Dominicana. (2012). Los feminicidios en la República Dominicana (Resumen 2005-2011). Santo Domingo: Procuraduría General de la República.

Rodríguez, M. (2000). Una revisión ideológica, sistémica y de género. Santo Domingo: Centro de Investigación y Ciencias de la Familia-Universidad Católica de Santo Domingo.

Roudinesco, E. (2006). La familia en desorden. México: Fondo de Cultura Económica.

Sonkin, D. J. et al. (1985). The male batterer. A treatment approach. New York: Publishing Company.

Unicef (2012). Estado mundial de la Infancia. Barcelona: Unicef.

Organización Panamericana de la Salud (1998). Program on women health and development. Progress in the eradication of violence against women (PAHO). Washington: OPS.

Vergés, L. (2010). Agresores de mujeres no tienen cuadro psiquiátrico. Listín Diario (lunes 15 de noviembre). 


\section{Huberto Bogaert García}

Huberto Bogaert García es psicólogo clínico. Se graduó de doctor en Psicología (Ph.D) en la Universidad Católica de Lovaina y se formó como psicoanalista en la Escuela Belga de Psicoanálisis. Recibió el Diploma de Estudios Especializados en Psicoanálisis (D.E.A.) en la Universidad de París VIII, se graduó de licenciado en Filosofía en el Instituto Tomás Moro (Bélgica). Es egresado de la Facultad de Ciencias Económicas y Sociales de la Universidad Autónoma de Santo Domingo.

En la actualidad, ejerce la psicología clínica en consulta privada, y es profesor universitario. Es autor de Enfermedad mental, psicoterapia y cultura (1992); Los enigmas de la sexualidad femenina (1993); La transferencia. Una investigación clinica con el test de Rorschach (1999); y coautor de La neoprostitución infantil en República Dominicana (1994); ha publicado artículos sobre temas psicológicos en revistas nacionales y extranjeras.

E-mail: hubertobogaert@yahoo.com

Recibido: 15/10/2013 Aprobado: 10/10/2014 